\title{
A Rare case in Indians -- Berardinelli-Seip Congenital Lipodystrophy
}

\author{
Sucharita Datta, $\mathrm{MD}^{1,}$ Saumyen De, $\mathrm{MD}^{2}$, Isita Tripathy ${ }^{3}$ \\ ${ }^{1}$ Associate Professor, ${ }^{2}$ Assistant Professor, ${ }^{3}$ Post Graduate Trainee, Department of Pediatrics, Nilratan Sircar \\ Medical College, West Bengal University of Health Sciences, India
}

\begin{abstract}
Berardinelli-Seip Congenital Lipodystrophy( BSCL) also called Congenital Generalised Lipodystrophy $(C G L)$ is a very rare autosomal recessive metabolic syndrome with a prevalence of less than 1 case in 12 million $^{1}$. About 200 cases have been reported so far world-wide ${ }^{2}$. BSCL2, the more severe form of the disease with onset in the neonatal period or early infancy is present in Lebanon, Portugal, Norway and the Middle East with the world's highest prevalence in the Brazilian population of Portuguese descent ${ }^{3,4,5}$. However , reports from India are few and far between. We are therefore reporting a case of a 3 year old boy who presented wih features typical of the disease, characterised by generalised lipodystrophy, acromegaloid features, hepatosplenomegaly, involvement of the face with empty cheeks and hypertriglyceridemia along with mild mental retardation, penile enlargement and hirsutism. The boy had developed an obstructed Richter's hernia at 3 months of age for which he was operated and subsequently developed cirrhosis at 1 year of age. Other than only one report of a case of BSCL2 having developed cirrhosis as early as 4 months of age, ours is the second report of BSCL with early onset of cirrhotic changes in the liver by one year of age. Moreover, though there have been some reports of umbilical hernia associated with BSCL, ours is the first report of Richter's hernia developing at 3 months of age in a case of BSCL.
\end{abstract}

Keywords - Berardinelli-Seip Congenital Lipodystrophy, Liver cirrhosis, Richter's hernia

\section{INTRODUCTION}

Berardinelli-Seip Congenital Lipodystrophy ( BSCL) is a rare autosomal recessive metabolic disorder characterised by generalised loss of body fat ( including buccal pad of fat), muscular hypertrophy, hepatosplenomegaly, long extremities (hands and feet) and acromegalic appearance along with severe metabolic alterations in carbohydrate and lipid metabolism ( insulin resistance and its complications,viz., diabetes mellitus , dyslipidemia, hepatic steatosis and acanthosis nigricans).

Also called Congenital Generalised Lipodystrophy, BSCL1 was first described by Beradinelle in 1954 in a 2 year old boy in Brazil and later confirmed by Seip in Norway in 1959 in 3 patients, two of whom were brothers.

BSCL1, with affected gene on chromosome 9q13 prevalent in African-American population is milder variety presenting in the second or third decade of life ${ }^{6}$. BSCL2 with the mutant gene on chromosome 11q13 is more severe with onset in the neonatal period or early infancy, mild pscychomotor or mental retardation and a somewhat more prevalence wordwide than that of BSCL1 ${ }^{7}$.

BSCL3 has been recently studied by Kim et al in an individual born of consanguineous marriage resulting from the Glu 38X mutation in homozygous in the Caveolin-1 (CAV 1) gene .

In this report, we present a 3-year old boy, born of a second degree consanguineous marriage, affected by BSCL, with symptoms in early infancy and a full-blown clinical picture with development of hepatic cirrhosis by 1 year of age.

\section{CASE REPORT}

A 13 month- old male child born of second degree consanguineous marriage was admitted in our department with the chief complaints of generalised muscular hypertrophy since 2 months of age and progressively increasing abdominal size since 4 months of age. The child's mother also observed that since 6 months of age he had been progressively developing pecuiliar facial features ( such as empty cheeks, profuse facial hair and forward projecting lower jaw). (Fig-1)\& (Fig-2)The mother also gave a history of the child having undergone surgery for an obstructed right sided Richter's hernia at 3 months of age. But she gave no history of muscle weakness or fatigue,respiratory difficulty, early morning sweating or convulsion and jaundice in her child. On general examination, the child exhibited typical acromegaloid facies with a prominent forward projecting jaw line (prognathism), loss of buccal pad of fat with empty cheeks, hypertrophied, prominent muscles involving all four extrimities and trunk giving the boy an athletic appearance and profuse facial hair.

Anthropometric measurement revealed a weight of $8 \mathrm{~kg}\left(3^{\text {rd }}-15^{\text {th }}\right.$ percentile of CDC growth chart) and a length of $77 \mathrm{~cm}\left(50^{\text {th }}\right.$ percentile of CDC growth chart). Motor and language development were appropriate for age. 
However, the child was falling short of expected adaptive and social communication skills. On systemic examination, the abdomen was hugely enlarged with the liver spanning $14 \mathrm{~cm}$ and $7 \mathrm{~cm}$ palpable below the right costal margin and the left lobe palpable $6 \mathrm{~cm}$ at the epigastrium. It was firm to feel with a well defined margin. The spleen was $3 \mathrm{~cm}$ enlarged along its long axis, firm and non-tender.

The child underwent detailed investigations to determine the cause of his symptoms beginning with:

Complete blood count- which was within normal limit.

Liver function test- showed a mild conjugated hyperbilirubinemia. (Total bilirubin:2.6 mg/dl; Direct $1.8 \mathrm{mg} / \mathrm{dl}$ ) deranged liver enzymes (ALT:268U/L; AST:244 U/L; Alkaline phosphatase: $528 \mathrm{U} / \mathrm{L}$ ).

Prothombin time was normal (12.8 sec; INR :1).

Tests for viral markers such as HBsAg, Anti HCV IgG and Anti HAV total IgG and IgM were nonreactive. Thyroid profile showed a borderline rise in levels of serum T3: $2.3 \mathrm{ng} / \mathrm{ml}$ (Normal $0.65-1.85 \mathrm{ng} / \mathrm{ml}$ ) and T4 $: 11.8 \mathrm{mg} / \mathrm{dl}(\mathrm{N}: 4.2-10.8 \mathrm{mg} / \mathrm{dl})$ and a TSH in the low normal range ie. $1.6 \mu \mathrm{IU} / \mathrm{ml}(\mathrm{N}: 0.4-6 \mu \mathrm{IU} / \mathrm{ml})$.

Serum calcium was $11.48 \mathrm{mg} / \mathrm{dl}(\mathrm{N}: 8.8-10.8 \mathrm{mg} / \mathrm{dl})$ and serum inorganic phosphorus $-4.56 \mathrm{mg} / \mathrm{dl}$ ( 3.8-6.5 $\mathrm{mg} / \mathrm{dl})$.

Liver biopsy revealed effacement of normal lobular architecture with multiple nodules, well demarcated by portal and periportal fibrous bands infiltrated by lymphonuclear cells. The nodules were composed of hepatocytes showing clear cytoplasm, vacuolated cytoplasm as well as eosinophilic cytoplasm in many areas. The nuclei were small and mostly centrally located. Nuclei with cytoplasmic vacuoles were present at places. Some of the liver cells showed eosinophilic inclusions. The Periodic acid-Schiff (PAS ) stain showed diastase resistant PAS positive material(Fig-3). The impression was that the features were compatible with Glycogen Storage Disease with development of cirrhosis.

But, before we could further proceed with the work-up, the child was lost to follow-up.. After a lapse of about 2 years the child was brought to our out-patient department for aggravation of his pre-existing complaints. On re-evaluating the child, we found that the generalised muscular hypertrophy involving the extremities had aggravated with additional pectoral muscle hypertrophy. The abdomen had further protruded and manifested a large umbilical hernia.Long hands and feet depicted acromegaloid features. The boy now manifested generalised hirsutism involving the trunk and bilateral upper and lower limbs. ( earlier presented with only facial hirsutism). Prominent veins were seen both in the upper and lower extremties ( phlebomegaly). The phallus was enlarged and hyperpigmented patchy areas (acanthosis nigricans) had appeared in the axilla and in the nape of his neck.

The child now weighs $15 \mathrm{~kg}\left(50^{\text {th }}-85^{\text {th }}\right.$ percentile in CDC growth chart), has a height of $88 \mathrm{~cm}$ (on $3^{\text {rd }}$ percentile in CDC growth chart). Language and motor development were appropriate for age with persistence of deficits in cognitive, adaptive and communication skills.

On systemic examination, we found a hugely distended abdomen with an enhanced liver span of $20 \mathrm{~cm}$ on admission. The liver wass palpable $14 \mathrm{~cm}$ below the right costal margin. It was firm and non-tender.The spleen was firm and non-tender and palpable $9 \mathrm{~cm}$ along the long axis. This time, we worked -up on the lipid profile which showed a normal serum cholesterol,of $176 \mathrm{mg} / \mathrm{dl}$ ( $\mathrm{N}:<200 \mathrm{mg} / \mathrm{dl}$ ), a normal LDL of $112 \mathrm{mg} / \mathrm{dl}($ $\mathrm{N}$ : $<130 \mathrm{mg} / \mathrm{dl})$ and a reduced $\mathrm{HDL}$ of $22 \mathrm{mg} / \mathrm{dl}(\mathrm{N}:<10$ years : $>40 \mathrm{mg} / \mathrm{dl})$ and a four fold rise in triglyceride level (TG: N: $<10$ years : $\leq 100 \mathrm{mg} / \mathrm{dl}$ ) to $393 \mathrm{mg} / \mathrm{dl}$. CPK was estimated to be $103 \mathrm{IU} / \mathrm{L}(\mathrm{N}: 5-130 \mathrm{IU} / \mathrm{L}$ ) . Serum uric acid was normal at $4.5 \mathrm{mg} / \mathrm{dl}(\mathrm{N}: 1.7-5.8 \mathrm{mg} / \mathrm{dl})$. Morning blood sugar was within normal limits- 75 $\mathrm{mg} / \mathrm{dl}$. (N: 70-110mg/dl).

2D doppler echocardiography was within normal limits.

At this point of time we realised that though the liver biopsy report suggested that the findings were compatible with glycogen storage disase, the boy's mature-looking face with acromegaloid features, generalised muscular hypertrophy, absence of hypoglycemia and hperuricemia and generalised hirsutism did not make the diagnosis of glycogen storage disease tenable. We were then in a real dilemma as to whether we were dealing wih a case of GSD or some other disorder that we don't encounter commonly.

After a vigorous search of literature, we found that the clinical features of the child and the laboratory investigations are in favour of a very rare disorder called Congenital Generalised Lipodystrophy (CGL) or Berardinelli -Seip Congenital Lipodystrophy (BSCL).

\section{Discussion}

We have confidently made a diagnosis of BSCL from the typical dysmorphology, the findings on clinical examination and the blood biochemistry ${ }^{9}$. There is medical literature providing some major and minor criteria, presence of which arouses a high index of suspicion. Presence of 3 established major or 2 major along with 2 or more minor criteria establishes the diagnosis ${ }^{9}$. Our child has 5 of the 6 listed major criteria, viz, i) lipoatrophy affecting both trunk and limbs giving the child an athletic appearance, ii) empty cheeks, iii) acromegaloid 
features such as prognathism and long hands ands and feet with secondary phlebomegaly, iv) hepatomegaly ( span $=20 \mathrm{~cm})$ and $\mathrm{v}$ ) elevated serum triglyceride $(393 \mathrm{mg} / \mathrm{dl})$. To determine the $6^{\text {th }}$ major criterion,i.e., insulin resistance, plasma insulin was not estimated as this phenomenon usually manifests in the second decade. The fact that he is just 3 years of age may be the reason for his normoglycemia. He, however, has the patches of acanthosis nigricans on the nape of his neck and axilla - a cutanous marker of tissue resistance to insulin. Of the 5 listed minor criteria he satisfies 3 criteria viz: i) mild mental retardation ii) penile enlargement, iii) hirsutism.

This child showed an absence of both metabolically active adipose tissue of the limbs and trunk as well as the adipose tissue with a mechanical function such as the buccal cavity, face, palms, soles and the scalp. One study has shown that the 'absence' of adipose tissue with a mechanical function in the palms of the hands, soles of the feet and scalp was seen only in individuals affected with alterations in the BSCL2 gene, whereas the adipose tissue with a mechanical function appeared well preserved in these regions in the BSCL1 group.

The acromegalod features in this child such as prognathism and long hands and feet can be attributed to insulin-like growth factor (IGF ) hypersecretion leading to anabolic processes with rapid skeletal maturation. The generalised muscular hyprtrophy, thereby giving the child an athletic appearance is due of storage of abundant muscle glycogen and triglycerides accentuated by the anabolic processes due to hypermetabolism which is an important characteristic of $\mathrm{BSCL}^{1,10}$. Hepatomegaly may be the result of the anabolic processes operative in BSCL causing organomegaly further compounded by hepatic steatosis and abundant liver glycogen. Our child had already developed cirrhotic changes in the liver by 1 year of age. This is the second report of cirrhosis developing in BSCL at such an early age, the first report being that of a 4 month old infant with BSCL developing liver cirrhosis. The liver biopsy report of our patient though gave an impression of a diagnosis compatible with GSD, the comment of the PAS stain showing PAS positive diastase resistant material was a matter for debate. On searching up literature on BSCL, we found that according to a proposed theory, since a limited amount of triglycerides can be stored because of the absence of fat, the excess triglycerides accumulate in the liver and skeletal muscle. This lipid deposition may account for the diastase resistant PAS positive material found in the liver in our case.

In this disorder, the major deficiency in lipid metabolism lies in the inability of adipocytes to store fat, probably due to abnormal functioning of glucose transporters (GLUT1-7) found in adipose, musculoskeletal and cardiac cells. Consequently, intracellular levels of glycerol are low, hampering the storage of free fatty acids in the form of triglycerides. Nevertheless, the metabolic use of lipids and hepatic lipogenesis via carbohydrates are active, though limited.So, the excess glucose is preferably stored in the form of glycogen as observed in liver and muscle biopsies.

The splenomegaly may be explained as part of a general increase in lymphoid tissue or deposition of circulating lipids or portal hypertension.

A slight resistance to insulin is seen during childhood with a rapid increase in insulin resistance between 8 and 10 years of age with established diabetes at 12 years of age. Our child of only 3 years was normoglycemic. Plasma insulin though not measured may probably have been normal or slightly higher as the presence of acanthosis nigricans ( manifested as hyperkeratotic and hyperpigmented patches at the nape of the neck and axilla in our case) is closely associated with insulin resistance. Insulin generally acts on fibroblasts and keratinocytes and its increased concentration leads to an increased growth, metabolism and proliferations of epidermal cells, clinically expressed in the form of acanthosis nigricans.

The child showed precocious phallic enlargement- evidently, a part of organomegaly.

As genetic and mutational analysis were beyond our scope, we have tried to strike a co-relationship between the phenotype and the likely genotype. As BSCL1 mutations are found only in individuals of African origin $^{11}$, this gene mutation found on chromosome 9q34 is unlikely in our patient. BSCL1 is the milder variety, presenting in the second or third decade of life. Our patient presented in early infancy, with Richter's hernia by 3 months of age, history of which goes in favour of BSCL2. The mild mental retardation observed in our patient is found in $78 \%$ of BSCL2 according to one study, while only $10 \%$ of BSCL1 patients showed significant intellectual deficiency ${ }^{11}$. The fact that Seipin (BSCL2) gene is highly expressed in the central nervous system may be the cause for variability of intellectual deficit observed among individuals affected by a BSCL2 mutation $^{3}$.

The absence of adipose tissue with a mechanical function in the palms of the hands, soles of the feet, scalp and face of our patient was seen only in indivisuals affected with BSCL2 mutations according to one study, whereas the adipose tissue with a mechanical function--- which is relatively inert metabolically and performs protection and deadening functions against shock and friction--- appeared well preserved in these regions in the BSCL1 group of the same study.

Recently Kim et al studied 4 affected individuals that did not have mutation in the Seip (BSCL2) or AGPAT2 (BSCL1) genes and found the Glu38X mutation in homozygous in Caveolin1 (CAV1) gene of an individual born to a consanguineous marriage. CAV-1 is the key structural component of the plasma membrane and is thought to play an important role in insulin signalling and lipid homeostasis ${ }^{12}$. Our child with BSCL is also a 
product of a second degree consanguineous marriage. Individuals with CAV1 gene mutations are classified as BSL3. However, this child is unlikely to belong to the BSCL3 group, as a mandatory prerequisite in this group is an elevated CPK which was normal in this case.

Taking the above aspects into consideration, this child's Indian origin, clinical profile and biochemical markers are in favour of a most probable diagnosis of BSCL2.

Diet is the most important aspect of management in this disorder. They should eat an extremely low fat diet with substitution of eucaloric medium chain triglycerides for long chain fatty acids ${ }^{1}$.Metformin with or without insulin is the chosen drug for treatment as it reduces the appetite and hepatic steatosis. Recombinant leptin therapy is still in the experimental stage ${ }^{13}$.

As the recurrence risk is $25 \%$, chorionic villus sampling at 9-12 weeks is recommended for prenatal diagnosis in at-risk patients. The prognosis is poor. Most patients succumb in the second or third decade of life due to complications related to diabetes, atherosclerosis or chronic liver failure.

\section{CONCLUSION}

Our child with BSCL, apart from the characteristic dysmorphology and metabolic abnormalities of this syndrome, was rather exceptional in that he had developed an obstructed Richter's hernia at 3 months of age and had developed cirrhotic changes in the liver by one year of age. He also showed the earliest signs of insulin resistance ( acanthosis nigricans ) by 3 years of age, albeit in the background of normoglycemia.

Different mutations can lead to different clinical manifestations. So, as soon as such children express the first signs of lipoatrophy and the disease is identified by clinical and metabolic parameters, it is necessary to go for genotyping and continuous monitoring from childhood until adulthood - for observing the evolution of the disease and adopting preventive measures for the control of metabolic complications. These measures will not only provide a better quality of life, but hopefully prolong the survival of individuals affected by BSCL. ${ }^{3}$

\section{REFERENCES.}

[1] Garg A. Acquired and inherited lipodystrophies. New Engl J Med 2004;350: 1220-34

[2] Garg A. Lipodystrophies. Am J Med 2000; 108: 143

[3] Gomes KB, Pardini VC, Fernandes AP. Clinical and molecular aspects of Berardinelli Seip Congenital Lipodystrophy (BSCL). Clin Chim Acta 2009;402:1-6

[4] Gomes K, Fernandes A, Ferreira A, et al. Mutations in the seipin and AGPAT2 genes clustering in consanguineous families with Berardinelli-Seip congenital lipodystrophy from two separate geographical regions of Brazil. J Clin Endocrinol Metab 2004:89:357-61.

[5] Gomes K, Pardini V, Ferreira A, Fonseca C, Fernandes A. Founder Effect of the 669insA mutation in BSCL2 gene causing Berardinelli-Seip Congenital Lipodystrophy in a cluster from Brazil. Ann Hum Genet 2007;71:729-34

[6] Garg A, Wilson R, Barnes R, Arioglu E, Zaidi Z, Gurakan F, et al. A gene for congenital generalized lipodystrophy maps to human chromosome 9q34. J Clin Endocr Metab 1999; 84: 3390-3394.

[7] Magre J, Delepine M, Khallouf E, Gedde-Dahl T Jr, Van Maldergem L, Sobel E, et al. Identification of the gene altered in Berardinelli- Seip congenital lipodystrophy on chromosome 11q13. Nature Genet 2001; 28: 365-370

[8] Kim C, Delepine M, Boutet E, et al. Association of a homozygous nonsense caveolin-1 mutation with Berardinelli-Seip Congenital Lipodystrophy. J Clin Endocrinol Metab 2008;93:1129-34.

[9] [Maldergem Van L. Berardinelli-Seip congenital lipodystrophy. Orphanet encyclopedia. November 2001. Available from: URL: http://www.orpha.net/data/patho/GB/uk-berad.pdf.

[10] Seip M, Trygstad O. Generalized Lipodystrophy, Congenital and Acquired (Lipoatrophy). Acta Paediatr Sppl 1996;413:2-28.

[11] Van Maldergem L, Magré J, Khallouf T, et al. Genotype-phenotype relationship in Berardinelli-Seip congenital lipodystrophy. $J$ Med Genet 2002;39:722-33.

[12] Kim C, Delepine M, Boutet E, et al. Association of a homozygous nonsense caveolin-1 mutation with Berardinelli-Seip Congenital Lipodystrophy. J Clin Endocrinol Metab 2008;93:1129-34.

[13] Beltrand, Beregszaszi M, Chevenne D, et al. Metabolic correction induced by leptin replacement treatment in young children with Berardinelli-Seip congenital lipoatrophy. Pediatrics 2007;120:291-6.

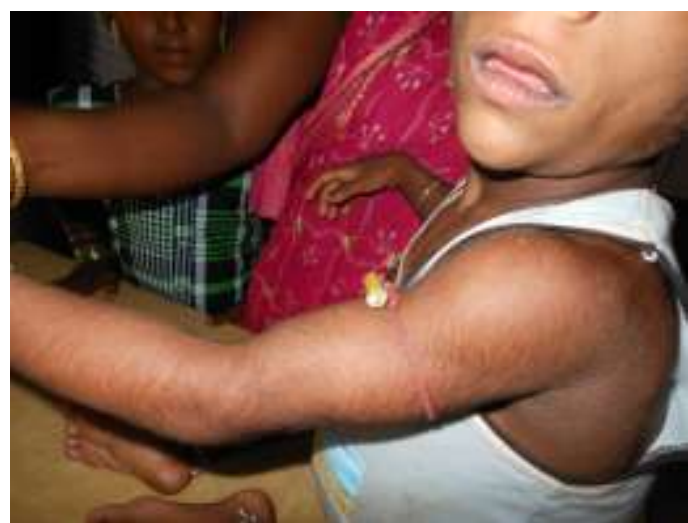

(Fig-1)3 year old boy showing empty cheeks, prognathism, hypertrophied muscles and hirsuitism 


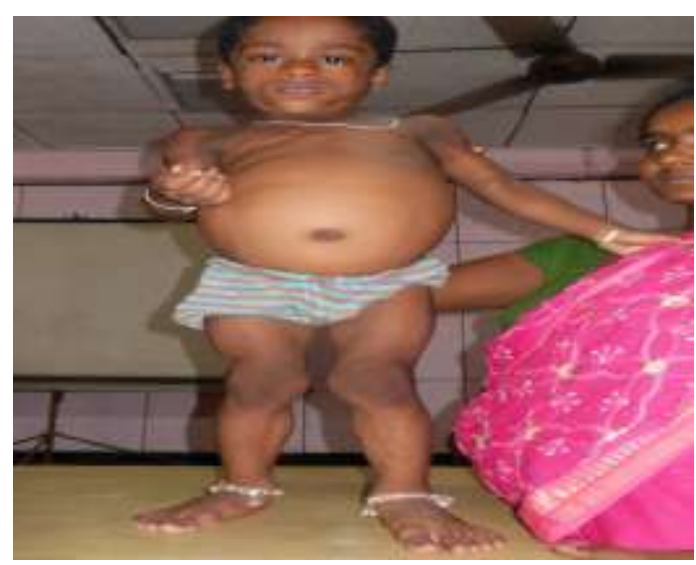

(Fig-2)Front view of the child showing a protruded abdomen with generalised muscle hypertrophy involving all 4 limbs, acromegaloid features of the face, hands and feet and acanthosis nigricans around both shoulders

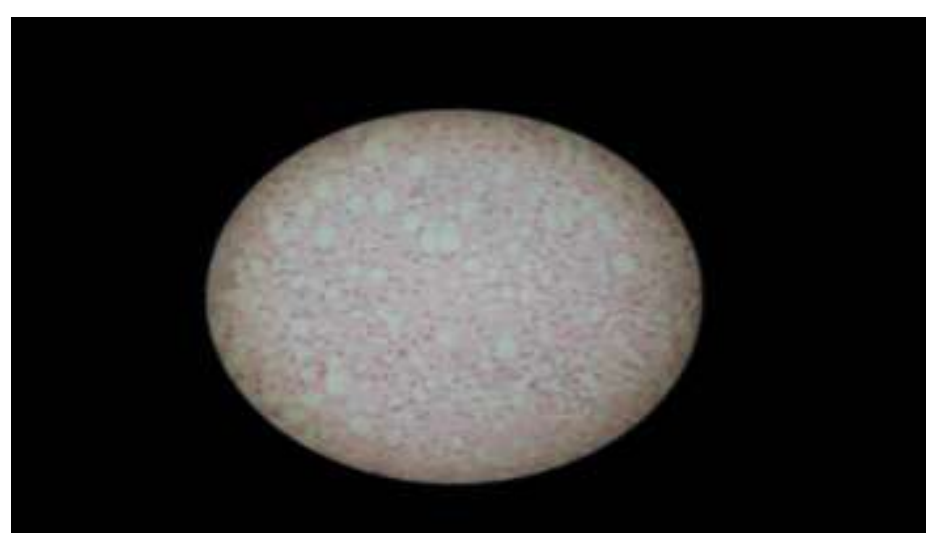

(Fig-3)Liver biopsy showed features compatible with glycogen storage disease with evidence of cirrhosis, but diastase resistant PAS positive materials were found 